\title{
CARACTERIZACIÓN BROMATOLÓGICA, FISICOQUÍMICA MICROBIOLÓGICA Y REOLÓGICA DE LA PULPA DE BOROJÓ (Borojoa patinoi Cuatrec)
}

\section{CHARACTERIZATION BROMATOLOGICAL, PHYSICO-CHEMICAL, MICROBIOLOGICAL AND RHEOLOGICAL IN BOROJO PULP (Borojoa patinoi Cuatrec)}

\author{
${ }^{\bullet}$ Raúl Díaz Ocampo르 Luis García Zapateiro², José María Franco Gómez ${ }^{3}$, Christian Vallejo Torres ${ }^{1}$ \\ ${ }^{1}$ Carrera de Ingeniería en Alimentos. Facultad de Ciencias Pecuarias, Universidad Técnica Estatal de Quevedo, \\ km 7 vía Quevedo - El Empalme, C. P. 73. Mocache,Los Ríos, Ecuador. ${ }^{\bullet}$ rauldiaz63@gmail.com \\ ${ }^{2}$ Grupo de Investigación Ingeniería de Fluidos Complejos y Reología de Alimentos (IFCRA) \\ Universidad de Cartagena. Facultad de Ingeniería. Cartagena de Indias, Colombia. \\ ${ }^{3}$ Departamento de Ingeniería Química. Facultad de Ciencias Experimentales. Campus de "El Carmen". \\ Universidad de Huelva. 21071 Huelva, España.
}

\section{Abstract}

RESUMeN

$\mathrm{E}$ n este estudio se presenta la caracterización bromatológica, fisicoquímica, microbiológica y reológica de la pulpa fresca de borojó (Borojoa patinoi Cuatrec), que son de interés en el procesamiento industrial. Se analizaron muestras de la pulpa del fruto intacto de borojó, proveniente de la provincia de Los Ríos (Ecuador), el rendimiento de la pulpa fue del 82.3\% respecto del total de la fruta. La pulpa presenta un bajo $\mathrm{pH}$, y tiene niveles importantes de carbohidratos y calcio. La pulpa de borojó obtenida fue de óptima calidad microbiológica, con recuentos de microorganismos que están dentro de los rangos permitidos según la normativa. Se realizó la caracterización reológica mediante ensayos de flujo en estado estacionario y ensayos oscilatorios de la pulpa de borojó a diferentes temperaturas. La pulpa de borojó presentó un comportamiento no newtoniano de carácter pseudoplástico, con un comportamiento más elástico que viscoso similar a un gel. Los resultados de los ensayos de flujo y oscilatorios muestran que la temperatura influyó en el comportamiento reológico de la pulpa de borojó.

Palabras clave: Borojó (Borojoa patinoi Cuatrec), pulpa, bromatología, microbiología, reología.
$\mathrm{T}$ his research present the characterization bromatological, physico-chemical, microbiological and rheological borojo fresh pulp (Borojoa patinoi Cuatrec), which are of interest in the industrial processing of this pulp. Samples of intact borojo pulp were analyzed. These samples are coming from Los Ríos Province (Ecuador), the amount of pulp obtained was $82.3 \%$ respecting the whole fruit. The pulp presents a low $\mathrm{pH}$ and an important level of carbohydrates and calcium. The borojo pulp obtained was microbiological high quality, with microorganism counts inside the ranges normatively allowed. Rheological behavior is characterized by oscillatory and flow tests at different temperatures. The results showed that the borojo pulp behaves as a non-Newtonian pseudoplastic fluid, where the viscosity has a more elastic behavior than viscous similar to a gel. The results of the tests oscillatory and flow shows that temperature influenced on rheological behavior of borojo pulp.

Key words: Borojo, pulp, bromatological, microbiology, rheology.

\section{INTRODUCCIÓN}

A ctualmente existe una demanda de alimentos más saludables y un interés comercial por frutas con alto poder nutricional y propiedades nutraceúticas. En este contexto, las frutas amazónicas han ganado un interés notable en el mercado de productos exóticos frescos y procesados, entre ellas el borojó (Borojoa patinoi Cuatrec) destaca por sus importantes propiedades

Recibido: 14-Febrero-2012. Recibido en forma corregida: 23-Abril-2012. Aceptado: 22-Junio-2012.

Publicado como ARTÍCULO CIENTÍFICO en Ciencia y Tecnología 5(1): 17-24. 2012 alimenticias, excelentes condiciones de adaptación y por su potencial económico, constituyéndose en importante insumo para la industria alimentaria.

El B. patinoi es una especie arbórea perteneciente a la familia Rubiáceae, originaria de América Tropical, restringida al centro de la zona del Ecuador climático, el fruto es una baya carnosa de 7 a $12 \mathrm{~cm}$ de diámetro, 
que en sus primeros estadios es de color verde clara y al madurar se torna parda rojiza; tiene un mesocarpio carnoso con sabor aromático y muy perfumado (Giraldo et al., 2004), los frutos tienen un peso promedio de $740 \mathrm{~g}$, constituidos por pulpa, semilla y cáscara, con frecuencia, las semillas llegan a constituir hasta un 10\% del peso del fruto (Jaramillo et al., 2005).

Tradicionalmente las comunidades indígenas asentadas en la zona amazónica de donde proviene el B. patinoi, lo han utilizado como alimento y producto curativo; entre los usos medicinales se menciona que es satisfactorio para cicatrizar heridas, controlar el azúcar en la sangre o la hipertensión. Estas comunidades lo consideran un alimento con alto valor nutritivo por su alto contenido en minerales, más que por el sabor de la fruta. (Hollihan, 2004).

Este fruto es comúnmente utilizado en América del Sur, en productos como jugos, pulpas y mermeladas, por su alto contenido de sólidos y bajo $\mathrm{pH}$. A pesar de ser una fruta poco perecedera y de alta disponibilidad en su medio natural, el $B$. patinoi es un producto de difícil manejo debido a su consistencia y peso, por tal razón, se hace necesario emplear diversos métodos para la manipulación, entre estos el despulpado. La pulpa de $B$. patinoi es altamente energética y nutritiva, con alto contenido de sólidos solubles, proteínas, aminoácidos y fósforo (Mosquera et al., 2005).

La pulpa es de color café, ácida y densa. $\mathrm{Su}$ nombre proviene del dialecto citara y significa "árbol de cabeza colgante" (Millán et al., 2010), es el producto carnoso y comestible de la fruta, obtenido por procesos tecnológicos adecuados de buenas prácticas de postcosecha y manufactura (INEN, 2008).

La pulpa de la fruta madura de $B$. patinoi puede conservarse al ambiente o en nevera, y en envases herméticos por hasta seis meses sin necesidad de aditivos. La parte comestible del B. patinoi puede ser procesada en forma de pulpa o de hojuelas deshidratadas, la pulpa es muy adhesiva, por lo que deben utilizarse envases de plástico o de vidrio. Se han estudiado algunas características de las propiedades funcionales de la pulpa obtenida mediante el secado por aspersión y el efecto de la maltodextrina en la estabilidad de la pulpa de $B$. patinoi seca por liofilización (Mosquera et al., 2010).

En la superficie del fruto o de la pulpa se desarrollan frecuentemente micelios de hongos, probablemente Aspergillus y Penicillium, la cual debe ser prevenida mediante un buen lavado y desinfección antes del despulpado. En el fruto estos hongos no causan daño, porque no pasan el pericarpio (Mejía, 1984).

El contenido de sólidos solubles, la firmeza de los frutos, el color de la pulpa, el contenido de fibra de raíces y tallos, el grado de turgencia que presentan al ser procesados, son algunos de los atributos que inciden en la calidad industrial de la materia prima.

El B. patinoi se encuentra en una etapa de introducción en Ecuador ya que es una planta que se desarrolla en condiciones óptimas en las regiones tropicales húmedas, es bastante resistente a plagas y enfermedades y de fácil adaptación. La explotación se divide principalmente a nivel de monocultivo, en cultivo asociado con otras especies o la producción de árboles dispersos, empezando a mostrar gran interés los productores agrícolas para domesticarlo y producirlo intensivamente.

Debido a la aceptación y comercialización cada vez más creciente de los productos derivados del $B$. patinoi, se requiere una información científica más amplia sobre las características de esta fruta, por ello se considera interesante el estudio del comportamiento reológico de la pulpa de esta fruta para su caracterización y utilización en el desarrollo de nuevos productos. Las características reológicas de un fluido complejo es uno de los criterios esenciales en el desarrollo de productos en la industria alimentaria, en el control de calidad, así como en la comprensión y caracterización de atributos texturales. Las propiedades reológicas de los distintos alimentos, normalmente tienen gran importancia en el procesado, transporte y almacenamiento (Steffe, 1996).

Numerosas investigaciones en reología de alimentos confirman la variedad de comportamientos de flujo encontrados en sistemas alimentarios. Los fluidos no newtonianos exhiben un comportamiento de flujo que depende de la velocidad de cizalla y, generalmente, del tiempo, como consecuencia de cambios estructurales (Steffe, 1996). En el caso de fluidos pseudoplásticos, la viscosidad aparente desciende a medida que aumenta la velocidad de cizalla (Muller, 1973).

Por otra parte, la mayoría de los alimentos microestructurados muestran características viscoelásticas. Una forma frecuente de caracterizar el comportamiento reológico de alimentos en la región de viscoelasticidad lineal es mediante ensayos en cizalla oscilatoria (Steffe, 1996), donde se aplica una deformación oscilatoria de pequeña amplitud sobre el material y se registra la respuesta del esfuerzo, o viceversa, así como el ángulo de fase entre el esfuerzo cortante y la deformación. Este tipo de ensayos permiten determinar la relación entre las componentes elásticas y viscosas y caracterizar el tipo de material de acuerdo a la evolución de los módulos de almacenamiento, G', y pérdidas, G', que representan el comportamiento elástico y viscoso, respectivamente (Barbosa-Cánovas et al., 1993).

En este trabajo se aborda el estudio de las propiedades y características bromatológicas, físicoquímicas, microbiológicas y reológicas de la pulpa 
fresca de B. patinoi, como un aporte a la ciencia y la caracterización de esta fruta de origen amazónico.

\section{Materiales y métodos}

\section{Materia prima}

E tamaño de las muestras de pulpa fresca para los ensayos de caracterización bromatológica, físicoquímica, microbiológica y reológica fue de $3 \mathrm{~kg}$. Los lotes se obtuvieron de fincas aledañas al cantón Mocache, Provincia de Los Ríos (Ecuador).

$\mathrm{Se}$ escogieron al azar frutos maduros visiblemente sanos, se transportaron a los Laboratorios de Bromatología de la Universidad Técnica Estatal de Quevedo y de la Escuela Politécnica Nacional. La materia prima se pesó, se lavó para eliminar materiales extraños, los frutos se cortaron en mitades longitudinales y se realizó el despulpado de forma manual eliminándose la corteza y semillas. Las muestras fueron evaluadas por duplicado.

\section{Caracterización bromatológica y fisicoquímica}

Los análisis bromatológicos y fisicoquímicos se realizaron en base seca por duplicado, utilizando métodos normalizados (AOAC, 2000):

Humedad. La humedad se determinó en una estufa de vacío Heraus a $70^{\circ} \mathrm{C}$ y $100 \mathrm{~mm} \mathrm{Hg}$, según el método 920.151.

Cenizas. Para su determinación se utilizó una mufla Lindberg a $525^{\circ} \mathrm{C}$, por el método 940.26 .

Extracto etéreo. Se determinó en un extractor de grasas con éter de petróleo tipo Goldfisch, marca Labconco, según el método 920.85 .

Proteínas. Se determinaron con un equipo Kjeltec 1002, marca Tecator, de acuerdo al método 2001.11.

Sólidos solubles. El contenido de sólidos solubles se determinó mediante lectura directa en un refractómetro Zeiss-Abbe a $20^{\circ} \mathrm{C}$, según el método 932.12.

Acidez total titulable. Se determinó como ácido málico, según el método 942.15.

pH. Se determinó $\mathrm{pH}$ por lectura directa en el pH-metro Orión, a $25^{\circ} \mathrm{C}$, según el método 981.12.

Fibra cruda. Fue determinada por hidrólisis y filtración, según el método 113 (ICC, 2001).

Carbohidratos totales. Se calculó teóricamente su valor de acuerdo al método descrito por Hart y Fisher (1991).

Valor energético. Se realizó el cálculo teórico según la Tabla de Composición de Alimentos de Centro América (INCAP, 2007).

Fósforo. Se determinó el contenido en fósforo mediante espectrofotometría (Ranganna, 1977).

Hierro y fósforo. Se determinaron por digestión en vía microondas y lectura en espectrofotómetro de absorción atómica (DECAB-EPN, 2007).

Los resultados de la caracterización bromatológica y fisicoquímica se presentan en forma de valor medio y desviación estándar.

\section{Evaluación microbiológica}

Las normas de pulpas de frutas recomiendan también la determinación del recuento de aerobios mesófilos, coliformes totales, hongos y levaduras. Los análisis microbiológicos se realizaron por duplicado, utilizando métodos normalizados (FDA-CFSANBAM):

Recuento total de aerobios. Se utilizó un medio de cultivo de Plate Count Agar, con incubación a $35^{\circ} \mathrm{C}$ durante 48 horas (FDA-CFSAN-BAM, 2001).

Recuento de coliformes fecales. Se utilizó un medio de cultivo de Agar Rojo Bilis Violeta, con incubación a $35^{\circ}$ C durante 24 horas (FDA-CFSAN-BAM, 2002).

Recuento de hongos y levaduras. Se utilizó un medio de cultivo de Diclorán D G18, con incubación a $25^{\circ} \mathrm{C}$, durante 7 días (FDA-CFSAN-BAM, 2001).

\section{Evaluación reológica}

Se realizaron ensayos de flujo estacionario obteniendo curvas de viscosidad $\eta$ a temperaturas de 0 , $10,25,40$ y $60^{\circ} \mathrm{C}$ en reómetros de esfuerzo controlado Rheostress RS-150 y MARS (Modulate Advanced Rheometer System) de ThermoHaake (KarlshureAlemania), en un rango de esfuerzos aplicados de 10-3,500 Pa, usando una geometría placa-placa de superficie rugosa de $35 \mathrm{~mm}$ y separación entre placas de $1.0 \mathrm{~mm}$.

En este estudio, previo a la medición, todas las muestras se dejaron en reposo durante 15 min para permitir la relajación de las mismas, la temperatura de las muestras se mantuvo constante a $20 \pm 0.1^{\circ} \mathrm{C}$, mediante un sistema Peltier para el control de temperatura.

Se utilizó el método dinámico para el estudio de las propiedades viscoelásticas de la pulpa de B. patinoi, se realizaron ensayos dentro del rango de viscoelasticidad lineal, en un reómetro de deformación controlada ARES de la firma TA Instruments (New Jersey-USA) a 25, 40 y $60^{\circ} \mathrm{C}$, en un rango de frecuencia comprendido entre 0.03 y $75.36 \mathrm{rad} \mathrm{s}^{-1}$, usando una geometría placaplaca ( $25 \mathrm{~mm}$ diámetro, separación de $1.2 \mathrm{~mm}$ ). Los parámetros viscoelásticos obtenidos fueron módulo de almacenamiento $\left(G^{\prime}\right)$, módulo de pérdida $\left(G^{\prime \prime}\right)$ y 
tangente de ángulo de fase ( $\tan \delta)$.

Previamente, se realizaron barridos de deformación a una frecuencia de $1 \mathrm{rad} \mathrm{s}^{-1}$, a fin de determinar el intervalo de viscoelasticidad lineal. La deformación límite del intervalo lineal se encuentra en la tan $\delta$ de $2-3 \%$. El barrido de frecuencia se aplicó a una deformación del $1 \%$

\section{Diseño de experimentos y análisis estadístico}

Se utilizó un diseño de experimentos completamente al azar, a todos los datos generados de las determinaciones se les realizó un análisis de varianza (ANDEVA) con un grado de confiabilidad del 95\%. Para analizar datos y crear gráficas se utilizó el programa OriginLab Pro 8.0 (Origin Lab. Corporation Northampton, MA 01060).

\section{Resultados Y Discusión}

$\mathrm{E}$ 1 balance de masa realizado, permitió establecer que se puede obtener un rendimiento promedio del $82.3 \%$ de pulpa, siendo el resto la parte no comestible del fruto (corteza y semillas).

\section{Composición bromatológica y fisicoquímica}

La composición bromatológica y fisicoquímica de la pulpa fresca de $B$. patinoi analizada se presenta en el cuadro 1.

Cuadro 1. Componentes bromatológicos y fisicoquímicos de la pulpa fresca de $B$. patinoi

\begin{tabular}{|c|c|}
\hline Componentes / Unidades & Resultados \\
\hline Humedad (\%) & $69.41 \pm 0.26^{2}$ \\
\hline Sólidos solubles ${ }^{\circ}$ Brix a $20^{\circ} \mathrm{C}$ & $32.00 \pm 0.96$ \\
\hline Cenizas $(\%)^{1}$ & $0.73 \pm 0.01$ \\
\hline $\mathrm{pH}$ & $2.93 \pm 0.09$ \\
\hline Acidez titulable (\% como ácido málico) & $2.60 \pm 0.05$ \\
\hline Carbohidratos totales $(\%)^{1}$ & $29.03 \pm 3.40$ \\
\hline Extracto etéreo $(\%)^{1}$ & $0.06 \pm 0.01$ \\
\hline Proteína $(\%)^{1}$ & $0.78 \pm 0.02$ \\
\hline Fibra cruda $(\%)^{1}$ & $3.50 \pm 0.14$ \\
\hline Fósforo $\left(\mathrm{mg} 100 \mathrm{~g}^{-1}\right)^{1}$ & $1.82 \pm 0.07$ \\
\hline Hierro(mg $\left.100 \mathrm{~g}^{-1}\right)^{1}$ & $0.69 \pm 0.09$ \\
\hline Calcio(mg $\left.100 \mathrm{~g}^{-1}\right)^{1}$ & $17.70 \pm 2.65$ \\
\hline Valor energético (Calorías) & $108.00 \pm 0.71$ \\
\hline
\end{tabular}

${ }^{1}$ Base seca

${ }^{2}$ Desviación estándar
De la caracterización bromatológica y fisicoquímica de la pulpa de $B$. patinoi se puede concluir que el contenido de humedad obtenido en la pulpa fresca de $B$. patinoi coincide con los valores reportados en la literatura: de 69\% (Mejía, 1984) y de 64.83\% (Mosquera, 2005). Este contenido en humedad favorece el procesamiento de néctar, vino y otros.

El porcentaje de sólidos solubles fue de $32^{\circ}$ Brix, igualmente, similar al publicado por Mejía (1984) para el mismo material (29 a $41^{\circ}$ Brix).

El contenido de cenizas de la muestra de pulpa de $B$. patino $i$ analizada, también concuerda aproximadamente con valores publicados de 0.8 a 1.2\% (Mejía, 1984) y de $0.801 \%$ (Sotelo et al., 2010). El porcentaje de cenizas está asociado al contenido mineral y en general depende del manejo agronómico del cultivo.

El pH obtenido es similar a los reportados por otros autores en la pulpa de esta fruta: de 2.8 a 3.0 (Mejía, 1984) y de 3.08 (Jaramillo et al., 2005). La pulpa de $B$. patinoi presenta valores inferiores a los hallados en frutos como el noni (Chan-Blanco, 2006), perteneciente también a la familia de las Rubiáceas. El pH es importante en el control del desarrollo de poblaciones de microorganismos, de la actividad de sistemas enzimáticos, en el proceso de clarificación de jugos y bebidas, en la estabilidad de los mismos y de otros productos elaborados a partir de pulpa de frutas como jaleas y mermeladas, cuya firmeza, color y sabor están determinados por la concentración de iones hidrógeno.

La acidez titulable de la pulpa de $B$. patinoi fue de 2.6 menor a los valores reportados por otros autores: de 3.0 (Mejía, 1984) y de 3.08 (Jaramillo et al., 2005).

El cálculo de carbohidratos totales en la pulpa de B. patinoi refleja un alto contenido de carbohidratos, superior a $13.86 \%$ reportado por Jaramillo et al. (2005). El contenido de carbohidratos totales influye notablemente en el sabor de las frutas y es uno de los factores intrínsecos que favorece el crecimiento de las poblaciones de bacterias y mohos propios de la microflora (Jay, 1992).

Se encontró en la pulpa de $B$. patinoi un contenido de extracto etéreo de $0.06 \%$, valor similar al reportado de $0.04 \%$ por Arango et al. (1986) e inferior al encontrado de $0.83 \%$ por Salamanca et al. (2010). En general las frutas y los vegetales contienen muy bajas concentraciones de contenido graso, aunque existen algunas excepciones, tales como el aguacate, aceitunas, y algunas nueces, que en promedio tienen $20 \%$ de lípidos (Badui, 1996).

El contenido proteico bajo de la pulpa de $B$. patinoi indica ser inapropiada como fuente proteica por ser inferior a los valores reportados en un rango de $0.8 \%$ a $1.3 \%$ por Mejía (1984) y de $1.75 \%$ (Mosquera, 2005). 
La pulpa de $B$. patinoi estudiada presentó un valor de fibra cruda, inferior al valor de $10 \%$ encontrado por Mejía (1984) y de $9.8 \%$ por Mosquera (2005).

Los resultados de calcio y hierro de la pulpa analizada se asemejan a los valores reportados por Mejía (1984) y Jaramillo et al. (2005).

La cantidad de fósforo, es inferior al valor de 160 mg $100 \mathrm{~g}^{-1}$ reportado por Mejía (1984) y $130 \mathrm{mg} 100$ $\mathrm{g}^{-1}$ (Mosquera, 2010). El valor energético de la pulpa de $B$. patinoi concuerdan con el valor reportado por Mejía (1984). Aunque el valor energético total de las frutas es menos importante que en otros alimentos, es destacable que el valor energético de la pulpa de B. patinoi de 108 Cal, es más alto que en pulpas de otras frutas amazónicas, como mamey, marañón y anona (Cavalcante, 1991). Se concluye que la pulpa fresca de $B$. patinoi presenta condiciones favorables para la industria alimentaria debido a su bajo $\mathrm{pH}$, nivel importante de carbohidratos y calcio.

\section{Características microbiológicas}

Las características microbiológicas de la pulpa fresca de $B$. patinoi se presentan en el cuadro 2 .

\section{Cuadro 2. Características microbiológicas (Unida- des Formadoras de Colonias por gramo de muestra) de la pulpa fresca de $B$. patinoi}

\begin{tabular}{lc}
\hline Microorganismo & Resultados \\
\hline Aerobios mesófilos & $<1 \times 10^{1}$ \\
Coliformes totales & $<1 \times 10^{1}$ \\
Hongos & 410 \\
Levaduras & 30 \\
\hline
\end{tabular}

$<1 \times 10^{1}=$ indica no desarrollo de colonias. Dilución 1:10

El recuento de aerobios mesófilos a partir del cual se perciben modificaciones en los atributos organolépticos del producto es de $10^{6} \mathrm{UFC}^{-1}$. En la pulpa evaluada el recuento es muy inferior $y$, en cualquier caso, no se detectaron alteraciones organolépticas. Por otra parte, los recuentos de hongos y levaduras que son indicadores del deterioro de frutas y vegetales están dentro de los rangos permitidos en la normativa ecuatoriana (INEN 2 337, 2008). Por los resultados microbiológicos obtenidos se puede afirmar que la pulpa fresca de $B$. patinoi fue de óptima calidad microbiológica.

\section{Caracterización reológica}

Ensayos en flujo estacionario. Se llevaron a cabo ensayos en flujo estacionario, a diferentes temperaturas, observando la variación de la viscosidad con la velocidad de corte. En la figura 1 se pueden observar las curvas de flujo viscoso de la pulpa de B. patinoi, en función de la velocidad de corte, a temperaturas comprendidas entre 0 y $60^{\circ} \mathrm{C}$.

Los resultados obtenidos a diferentes temperaturas de $0,10,25,40$ y $60^{\circ} \mathrm{C}$, demuestran un comportamiento pseudoplástico, ya que combinan características propias de los sólidos elásticos y de los líquidos, caracterizado por un descenso potencial de la viscosidad con la velocidad de corte (Muller, 1973).

Por otra parte, no se observan diferencias significativas de la viscosidad aparente con la temperatura, con valores de $\eta_{0}$ muy similares, en un rango de $4 \times 10^{6} \mathrm{~Pa} \mathrm{~s}$ a $5 \times 10^{6} \mathrm{~Pa}$ s. El anterior valor, es indicativo de la viscosidad del material en situaciones de reposo o movimientos sumamente lentos.

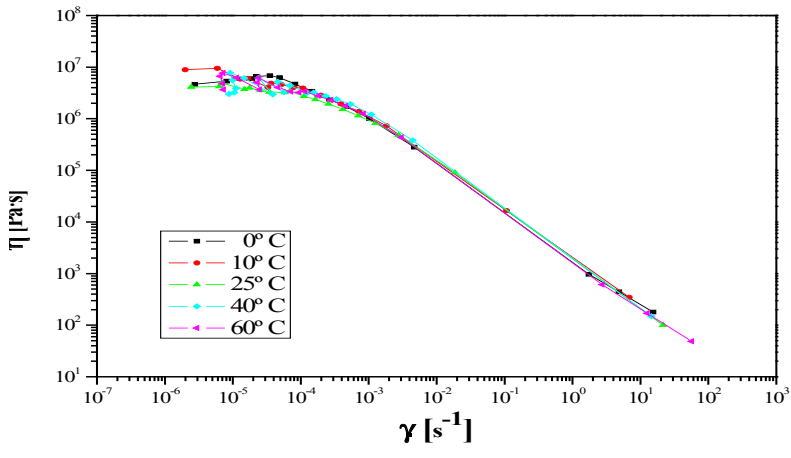

Figura 1. Curvas de flujo viscoso de la pulpa de $B$. patinoi a diferentes temperaturas.

La naturaleza de la pectina y la cantidad de partículas dispersas son determinantes principales en las propiedades de flujo de las pulpas. Este comportamiento pseudoplástico puede ser explicado por la ruptura de una estructura reticular de moléculas de polisacáridos durante el cizallamiento, tal y como describen Morris et al. (1981) y Bhandari et al. (2002). En un sistema reticular, la velocidad de ruptura de las interacciones moleculares existentes es superior a la velocidad de reformación de las mismas con el aumento de la velocidad de deformación. El resultado es una menor resistencia intermolecular al flujo $\mathrm{y}$, por tanto, una menor viscosidad.

Este mismo comportamiento se ha observado en diferentes alimentos derivados de la pulpa de otros 
frutos, tales como pulpa de guayaba (Harnanan, 2001), puré de guayaba (Vitali y Rao, 1982), en purés de mango, papaya y melocotón (Guerrero y Alzamora, 1998).

Ensayos oscilatorios. Se efectuaron por triplicados ensayos de barrido de deformación a $25^{\circ} \mathrm{C}$ para determinar la región viscoelástica lineal de la pulpa de B. patinoi como se muestra en la figura 2 .

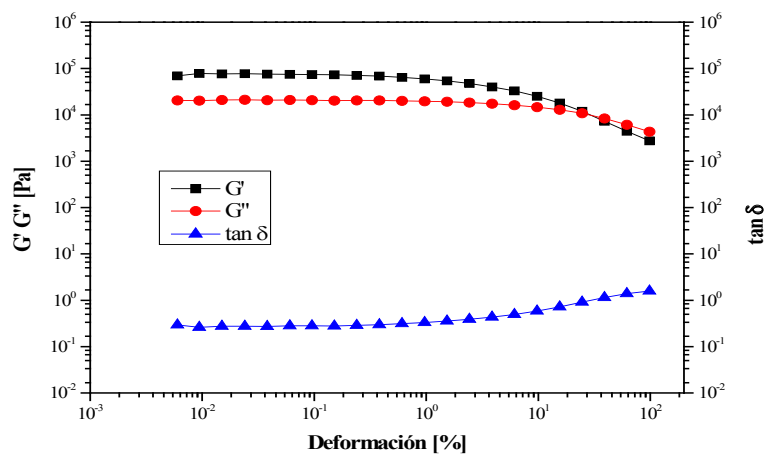

Figura 2. Módulo de almacenamiento (G'), módulo de pérdida (G”) y Tan $\delta$ en función de la deformación relativa de la pulpa de $B$. patinoi, a $1 \mathrm{~Hz}$ de frecuencia y $25^{\circ} \mathrm{C}$.

Donde se pudo establecer el rango lineal de la muestra para poder realizar los ensayos de barrido de frecuencia a diferentes temperaturas tomando como deformación relativa 1\%. El comportamiento de los módulos de almacenamiento G' (componente elástico) y pérdida G" (componente viscoso) se muestran en la figura 3.

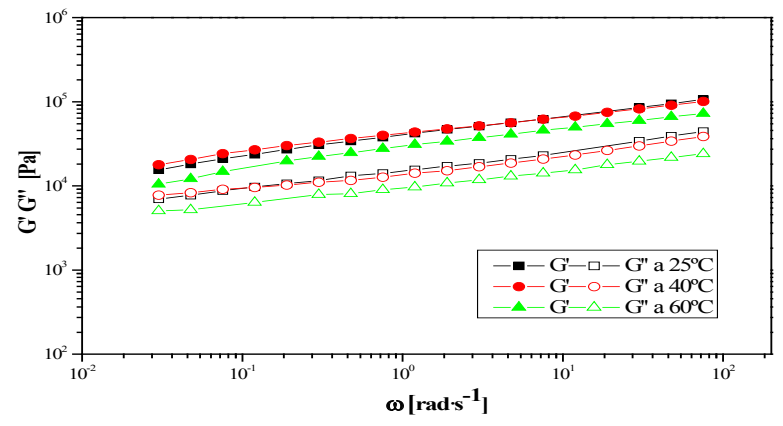

Figura 3. Módulo de almacenamiento (G') y Módulo de pérdida (G") de la pulpa fresca de B. patinoi en función de la frecuencia a diferentes temperaturas.

Como puede observarse en la figura 3 , los valores de G' son mayores que los de G', en todo el rango de frecuencias, presentando un comportamiento elástico, donde ambas funciones aumentan ligeramente con la frecuencia de forma casi paralela, similar al que se presenta en diversos alimentos como en las masas elaboradas con harinas de distintas variedades de trigos suaves (Magaña-Barajas et al., 2009).

En el cuadro 3 se muestran los valores de G', G" y de $\tan \delta$ en de la pulpa fresca de B. patinoi a una frecuencia de $30 \mathrm{rad} \mathrm{s}^{-1}$, a diferentes temperaturas. Se observa en todos los casos que $G^{\prime}>G^{\prime}$, lo cual demuestra un comportamiento elástico y a medida que se incrementa la temperatura disminuye el valor de G' y G', aumentando su comportamiento viscoso determinado con el valor de $\tan \delta$, por los resultados indica que es dependiente de la temperatura.

Cuadro 3. Parámetros viscoelásticos de la pulpa fresca de $B$. patinoi a una frecuencia de $30 \mathrm{rad} \mathrm{s}^{-1}$, a diferentes temperaturas

\begin{tabular}{cccc}
\hline $\mathbf{T}\left({ }^{\circ} \mathbf{C}\right)$ & $\mathbf{G}^{\prime}(\mathbf{P a})$ & $\mathbf{G}{ }^{\prime}(\mathbf{P a})$ & $\tan \boldsymbol{\delta}$ \\
\hline 25 & 94671 & 37282 & 0.396 \\
40 & 80995 & 29400 & 0.363 \\
60 & 54460 & 18186 & 0.342
\end{tabular}

La $\tan \delta$ se obtiene de la relación $\mathrm{G}^{\prime \prime} / \mathrm{G}^{\prime}$ y toma valores de 0 a 1 predominando un carácter elástico.

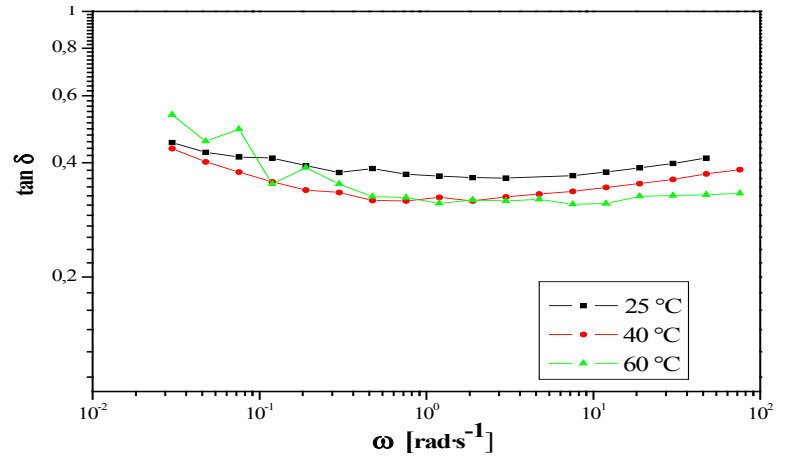

Figura 4. Tangente de ángulo de fase $(\tan \delta)$ en función de la frecuencia a diferentes temperaturas de la pulpa fresca de $B$. patinoi.

La $\tan \delta$ es una medida adimensional que compara la cantidad de energía perdida durante un ensayo oscilatorio con la cantidad de energía almacenada durante este periodo, e indica si predomina la propiedad elástica o viscosa (Mota y Ibarz, 2006). Se observa en la figura 4, que en función de la frecuencia y con el incremento de la temperatura, el valor de $\tan \delta$ de la pulpa fresca de B. patinoi presenta un ligero descenso, en cambio al aumentar el valor de la frecuencia también se incrementa ligeramente el valor de $\tan \delta$ para todas las temperaturas, lo que confirma para altas temperaturas el predominio de propiedades elásticas. 
Así, el comportamiento viscoelástico lineal mostrado por la muestra de pulpa de $B$. patinoi es similar al observado en otras pulpas de frutas, como mango (Pelegrine et al., 2002; Branco et al., 2003; Vidal et al., 2004 y Dak et al., 2007), guayaba (Ferreira et al., 2002 y Medina y Pagano, 2003), cereza (Da Silva et al., 2005), membrillo (Mota y Ibarz, 2006). Por otra parte, de forma análoga a lo que ocurre con la viscosidad, la respuesta viscoelástico de la pulpa de $B$. patinoi no está influenciada de forma significativa por la temperatura. Como se observa (Figuras 2 y 3), los parámetros viscoelásticos G', G” y $\tan \delta$ tuvieron un comportamiento similar disminuyendo ligeramente su valor con el incremento de la temperatura.

\section{Conclusiones}

El rendimiento de la pulpa obtenida fue del $82.3 \%$. La caracterización bromatológica y fisicoquímica de la pulpa fresca de B. patinoi permite catalogarla como ácida, con un buen nivel de calcio y constituye una fuente energética de importancia debido al alto contenido de carbohidratos.

Los recuentos de microorganismos permiten concluir que la pulpa fresca de B. patinoi es de óptima calidad microbiológica. El pH de 2.93 y la protección que brinda la corteza de la fruta, es un elemento o factor intrínseco que restringe el crecimiento de las poblaciones de microorganismos.

La pulpa de $B$. patinoi se comporta como un fluido no newtoniano pseudoplástico, donde la viscosidad disminuye con la velocidad de corte y presenta un comportamiento viscoelástico tipo gel, donde predomina su parte elástica.

La temperatura influyó en los comportamientos viscoso y viscoelástico de la pulpa de $B$. patinoi.

\section{Literatura CITADA}

AOAC. Association of Official Analytical Chemist. 2000. Official Methods of Analysis (17 th.Ed). Gaithersburg, MD, USA.

Arango, A. y T. Quijano. 1986. Estudio de los frutos de Borojoa Patinoi C. Revista Latin Química 17(3):167-169.

Badui, S. 1996. Química de los Alimentos. Longman/ Alhambra Mexicana. México. 645 p.

Barbosa-Cánovas, G. V., A. Ibarz, y M. Peleg. 1993. Propiedades reológicas de alimentos fluidos. Revisión Alimentaria 242:39-89.

Bhandari, P. N., R. S. Singlhal and D. D. Kale. 2002. "Effect of succinylation on the rheological profile of starch pastes". Carbohydrate Polymers 47:365-371.
Cavalcante, RV.1991. Frutas comestíveis da Amazônia. (5 Ed.). CEJUP, Museo Paraense E. Goeldi, Belém. 273 p.

Chan-Blanco, Y., F. Vaillant, A. Pérez, M. Reynesc, J. Brillouet y P. Brat. 2006. The noni fruit (Morinda citrifolia L.): A review of agricultural research, nutritional and therapeutic properties. Journal Food Compos Analysis 19(6):645-654.

Da Silva F., D. Guimaraes y C. Gasparetto. 2005. Rheology of acerola juice: effects of concentration and temperature. Ciência e Tecnología de Alimentos 25(1):121-126.

DECAB- EPN. 2007. Métodos analíticos. Escuela Politécnica Nacional. Quito. p. 1-9.

FDA-CFSAN-BAM (Food and Drug AdministrationCenter for Food Safety and Applied NutritionBacteriological Analytical Manual). 2001. Washington, USA. Chapter 3 Conventional Plate Count. p. 1-7.

FDA-CFSAN-BAM (Food and Drug AdministrationCenter for Food Safety and Applied NutritionBacteriological Analytical Manual). 2001. Washington, USA. Chapter 18. Yeast and Molds. p. 1-13.

FDA-CFSAN-BAM (Food and Drug AdministrationCenter for Food Safety and Applied NutritionBacteriological Analytical Manual). 2002. Washington, USA. Chapter 4. Enumeration of Escherichia Coli and the Coliform Bacteria. p. 1-14.

Ferreira, G., A. Melo, R. Silvestre y C. Gasparetto. 2002. Efeito da temperature no comportamento reológico das polpas de caju e goiaba. Revista Ciencias Exatas e Naturais 4(2):175-184.

Giraldo, C., L. Rengifo, E. Aguilar, D. Gaviria y A. Alegría. 2004. Determinación del sexo en borojó (Borojoa patinoi C.) mediante marcadores moleculares. Revista Colombiana de Biotecnología 6(2):9-14.

Guerrero, S. N. y S. M. Alzamora. 1998. Effect of pH, Temperature and Glucose Addition on Flow Behaviour of Fruit Purees: II. Peach, Papaya and Mango Purées. Journal of Food Engineering 37:77-101.

Harnanan, S. W. 2001. Effect of processing, preservation and storage on rheology of guava pulp. Journal of Texture Studies 32, 271-284.

Hart, F. L. y H. J. Fisher. 1991. Análisis Moderno de los Alimentos. Zaragoza. 8 p.

Hollihan, M. 2004. Estudio de mercado para el Borojó. CORPEI. Quito, Ecuador. 27 p.

I.C.C. 2001. International Association for Cereal Chemistry. 1. Standard 113.

INCAP (Instituto de Nutrición de Centroamérica 
y Panamá). 2007. Tabla de composición de alimentos de Centroamérica. ( $2^{\mathrm{a}}$ Ed.). Guatemala. p. $16-20$.

INEN. Norma Técnica Ecuatoriana 2337. 2008. Jugos, pulpas, concentrados, néctares, bebidas de frutas y vegetales. Requisitos. Quito, Ecuador.

Jaramillo, L., M. Arguello, A. Benítez y M. J. Borja. 2005. Ficha Técnica Biocomercio Sostenible en el Ecuador. CORPEI, Eco Ciencia y la iniciativa Biocomercio Sostenible-Ecuador. p. 15-16.

Jay, J. M., 1992. Modern Food Microbiology. (5th Ed.). Chapman \& Hall. p. 38 - 66; 149-176.

Magaña-Barajas, E., B. Ramírez Wong., L. C. PlattLucero., G. A. López Ahumada., P. I. Torres y D. I. Sánchez-Machado. 2009. Caracterización viscoelástica de masas de variedades de trigos suaves. Tecnol. Ciencia Ed. (IMIQ) 24(1):12-22.

Medina, M., y F. Pagano. 2003. Caracterización de la pulpa de guayaba (Psidiumguajava L.) tipo "Criolla Roja". Revista Facultad de Agronomía (LUZ) 20:72-86.

Mejía, M. 1984. Borojó. Fruta Ecuatorial Colombiana, Colombia Amazónica 1(2):89-106.

Millán, L., B. Cardona, J. A. Herrera, D. Arbeláez, y D. Gutiérrez. 2010. Análisis sensorial e instrumental (textura) a una salsa agridulce de borojó. Revista Lasallista de Investigación. Corporación Universitaria Lasallista Colombia 7(1):36-41.

Morris, E. R., A. Cutler, N. Ross-Murph, S. B. Rees y J. Price. 1981. Concentration and shearrate dependence of viscosity in random coil polysaccharide solutions. Carbohydrate Polimer 1:5-21.

Mosquera, L. H., H. A. Ríos y P. S. Zapata. 2005. Obtención de una materia prima con valor agregado mediante secado por aspersión a partir del fruto fresco de borojó (Borojoa patinoi C.) Revista Institucional Universidad Tecnológica del Chocó 11(23):5-10.
Mosquera, L. H., G. Moraga y N. Martínez-Navarrete. 2010. Effect of maltodextrin on the stability of freeze-dried borojó (Borojoa patinoi C.) powder. Journal Food Engineering 97(1):72-78.

Mota, A. y A. Ibarz. 2006. Comportamiento viscoelástico de pulpa de membrillo en función de la concentración de sólidos solubles. Revista Ciencia y Tecnología de los Alimentos. Campiñas 26(1):217-219.

Muller, H.G. 1973. An Introduction to food rheology. Heinemann. London. Cap. 6. p. 57-74.

Pelegrine, D.H., F. Silva y C. Gasperrato. 2002. Rheological behavior of mango and pineapple pulps. Lebensmittel-Wissenschaft und-Technologie 35(1):645-648.

Ranganna, S. 1977. Manual of Analysis of fruit and vegetable products. New Delhi. Cap.6. p.108109.

Salamanca, G., M. P. Osorio y L. M. Montoya. 2010. Elaboración de una bebida funcional de alto valor biológico a base de borojó (Borojoa patinoi). Revista chilena de nutrición 37(1):89.

Sotelo, I., N. Casas y G. Camelo. 2010. Borojó (Borojoa patinoi): Fuente de polifenoles con actividad microbiana. Revista Vitae 17(3):329-336.

Steffe, J. 1996. Rheological methods in food process engineering (2nd Ed.) East Lansing, USA. 382 p.

Vidal, J., D. Pelegrine y C. Gasparetto. 2004. Effect of the rheological behavior of mango pulp (Mangifera indica L-Keitt). Revista Ciencia y Tecnología Alimentaria 24(1):39-42.

Vitali, A. y M. A. Rao. 1982. Flow behavior of guava puree as function of temperature and concentration. Journal of Texture Studies 13:275289. 